\title{
Method for Improving Camouflage Image Quality using Texture Analysis
}

\author{
Kalyani V. Patil \\ Department of Electronics \\ SSVPS's B.S.D COE, Dhule \\ Maharashtra, India
}

\author{
K. N. Pawar \\ Professor \\ Department of Electronics \\ SSVPS's B.S.D COE, Dhule \\ Maharashtra, India
}

\begin{abstract}
Traditional evaluation method of camouflage texture effect is subjective evaluation. It's very tedious and inconvenient to direct the texture designing. In this project, a systemic and rational method for direction and evaluation of camouflage texture designing is proposed. Camouflage consists of things such as leaves, branches, or brown and green paint, which are used to make it difficult for anenemy to see military forces and equipment. A camouflage texture evaluation method predicated on SSIM (Weight structural homogeneous attribute) is given to access the effects of camouflage texture at first.Then nature image features between the camouflage texture and the background image are calculated to help direct the designing camouflage texture. In this project, we focus on the essential of the human visual system, and its relative significance of the different factors of affecting camouflage texture. The proposed method developed a computational vision model to evaluate the perceived differences between camouflage texture image and background image. And a variety of features, measuring thresholds for discriminating small changes in naturalistic images have been studied to direct the camouflage texture designing.
\end{abstract}

\section{Keywords}

Camouflage Image

\section{INTRODUCTION}

The role of images in present day communication has been steadily increasing. In This context the quality of an image plays a very important role. Different stages and multiple design choices at each stage exist in any image processing system. They have direct bearing on the quality of the resulting image. Unless we have a Quantitative measure for the quality of an image, it becomes difficult to design an ideal image processing system. Though subjective quality assessment is an alternative, it is not feasible to be incorporated into real world systems. Hence, objective quality metrics play an important role in image quality assessment.

In the last two decades a lot of objective metrics have been proposed to access image quality. The most widely adopted statistics feature is the Mean Squared Error (MSE). However, MSE and its variants do not correlate well with subjective quality measures because human perception of image distortions and artifacts is unaccounted for. MSE is also not good because the residual image is not uncorrelated additive noise. It contains components of the original image.A detailed discussion on MSE is given by Girod.

Camouflage texture is utilized to increment the survivability of soldiers and mission efficacy by obviating visual observation and other military sensors from detecting both the soldiers and their equipment's.Camouflage texture achieves the purposes by breaking the target contour and blending the target with the background. So it is very important to evaluate whether camouflage texture spots are consistent with the background in the shape, size, color and spatial distribution. Traditionally, the evaluation is conducted by groups of observers, who rate the effectiveness of camouflage texture. The authentic camouflage effects can be reflected in subjective evaluation, but some shortcomings and inhibitions, such as psychological factors of the observers and testing environment, in image quality subjective evaluation. It is very difficult in practical applications.

In this project, we focus on the essential of the human visual system, and its relative significance of the different factors of affecting camouflage texture. The proposed method developed a computational vision model to evaluate the perceived differences between camouflage texture image and background image. And a variety of features, measuring thresholds for discriminating small changes in naturalistic images have been studied to direct the camouflage texture designing.

Structural similarity based methods of image quality assessment claim to account for the fact that the natural image signal samples exhibit strong dependencies amongst themselves, which is ignored by most of these methods. Structural similarity based methods replace the Minkowski error metric with different measurements that are adapted to the structures of the reference image signal, instead of attempting to develop an ideal transform that can fully decouple signal dependencies.

However, Vision models, which treat visible distortions equally, regardless of their location in the image, may not be powerful enough to accurately predict picture quality in such cases. This is because we are known to be more sensitive to distortions in areas of the image to which we are paying attention than to errors in peripheral areas.

\section{CAMOUFLAGE TEXTURE EVALUATION METHOD}

\section{1) SSIM Texture Evalution Model}

Human visual model is based on knowledge of primary visual cortex, which recognizes a visual image by processing in parallel by channels or neurons with different optimal spatial frequencies. Based on human visual model, we propose a camouflage texture assessment framework to get the differences of background image and camouflage textures. Meanwhile, human eyes do not pay an equivalent attention to different regions in an image. In the assessment framework, different attention weights are applied to different regions of 
image. Fig.1 illustrates the camouflage texture assessment framework based on human visual model.

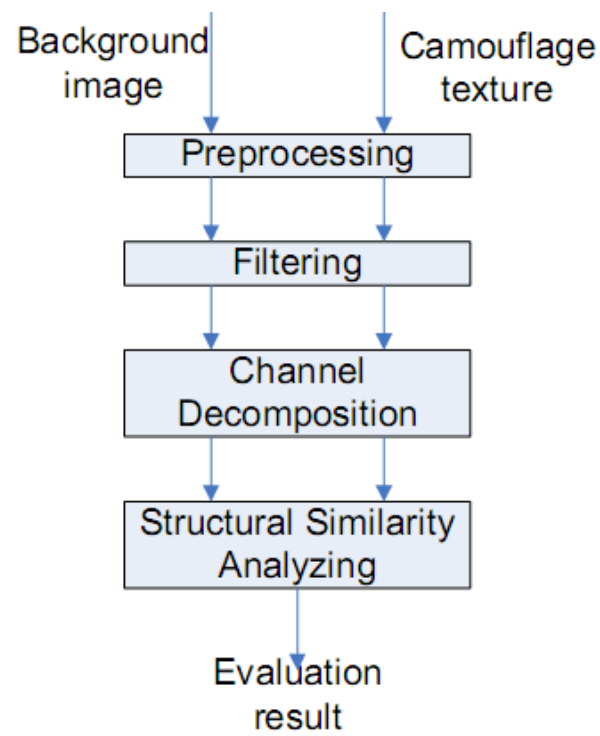

Fist, a variety of basic operations is performed to eliminate distortions from background image and camouflage texture. And a low-pass filter simulating the point spread function of the eye optics may be applied. Second, the luminance of camouflage texture and background image is compared as follows:

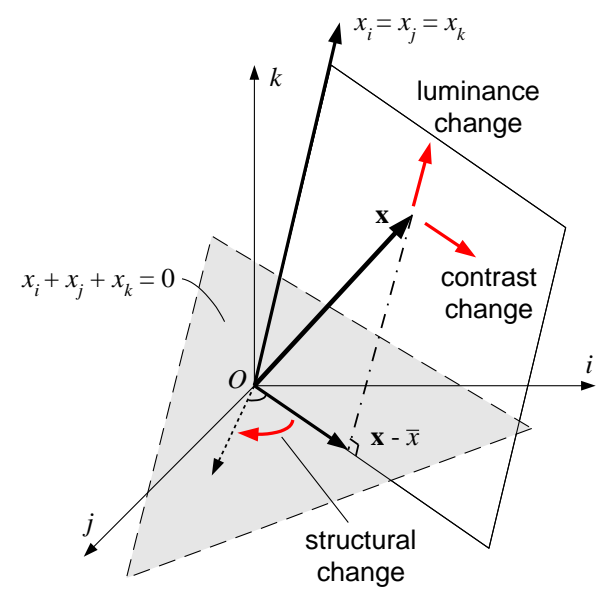

$$
l(x, y)=\frac{2 \mu_{x} \mu_{y}+C_{1}}{\mu_{x}^{2}+\mu_{y}^{2}+C_{1}}
$$

where $l(x, y)$ is the luminance comparison function of the mean intensity $\boldsymbol{\mu}_{x}$ and $\boldsymbol{\mu}_{y}$ of the camouflage texture $\mathrm{x}$ and background image y respectively. $\mathrm{C} 1$ is included to avoid instability, here we choose 6.5025 .

The contrast comparison function is given as follows:

$$
c(x, y)=\frac{2 \sigma_{x} \sigma_{y}+C_{2}}{\sigma_{x}^{2}+\sigma_{y}^{2}+C_{2}}
$$

where $\sigma_{x}$ and $\sigma_{y}$ is the stand deviation and we choose

\section{$C_{2} 58.5225$.}

We define the structure comparison function as follows:

$$
s(x, y)=\frac{2 \sigma_{x y}+C_{3}}{\sigma_{x} \sigma_{y}+C_{3}}
$$

where $\sigma_{x y}$ is the covariance and we choose $C_{3} 58.5225$.

\section{At last, the structural similarity (SSIM) index between $x$} and y can be drawn as

$$
\operatorname{SSIM}(x, y)=l(x, y) c(x, y) s(x, y)
$$

The camouflage texture is compared with each block of the background with same size to get the whole evaluation result by Weight-SSIM as follows:

$$
\operatorname{WSSIM}(X, Y)=\frac{1}{M} \sum_{j=1}^{M} w_{j} \operatorname{SSIM}\left(x_{j}, y_{j}\right)
$$

Where $\mathrm{Wj}$ is the weight of different block of background and $\mathrm{M}$ is the sum of the blocks. WSSIM is calculated three times on each pair of background image and camouflage texture for the red green and blue channels. The value will show the differences between the background image and the camouflage textures in RGB channels.

\section{2) Nature Image Feature Analysis}

Natural image signals are highly structuredtheir pixels exhibit strong dependence which carries important information about the structure of the objects in the visual scene. Structural features of natural images can be used to evaluate the effects of camouflage texture. We select some nature image features for directing the design of camouflage texture. The selected features are summarized in the following

- Average luminance:-

$$
\mu=\frac{1}{N} \sum_{i=1}^{N} x_{i}
$$

Where $\mathrm{N}$ is the sum of image pixel

- Standard Deviation :

$$
\sigma_{x}=\left(\frac{1}{N-1} \sum_{i=!}^{N}\left(x_{i}-\mu_{x}\right)^{2}\right)^{1 / 2}
$$

\section{- Correlation Length :}

Correlation length, which can be convenient from the image Fourier spectrum:

$$
r=10.0 / W
$$

where

$$
W^{2}=\frac{\sum\left(U^{2}+V^{2}\right)|F(U, V)|^{2}}{\sum|F(U, V)|^{2}}
$$

where $\mathrm{F}(\mathrm{V}, \mathrm{U})$ with $\mathrm{U}$ and $\mathrm{V}$ as(frequency) variables is the image Fourier spectrum. 


\section{- Texture Direction :}

which is the angle of the peak of the power spectrum

$$
P(u, v)=|F(U, V)|
$$

- Image Entropy :

$$
H(I)=-\sum_{i=0}^{L-1} \frac{c_{i}}{N} \log _{2}\left(\frac{c_{i}}{N}\right)
$$

Where $\mathrm{N}$ is the sum of image pixels, $\mathrm{L}$ is the sum of classes, and $\mathrm{c}$ is the pixels number of every class.

- By edge detection, the number of closed areas and the length of edge can be got. Background image and camouflage textures are treated with Canny edge detector [12] to extract the image edges. These features need to be normalized to ensure different Feature vector with similar effect. Gaussian normalization method is chosen because it can deal ultra-large or ultra-small element values. The function is given by:-

$$
Z=((X-\mu) /(3 * \sigma)+1) / 2
$$

where $\mu$ is the mean and $\sigma$ is thecovariance. These normalized features can measure the differences between camouflage texture and the background image, so according to the differences (e.g. luminance etc.) designers are able to improve the camouflage textures.

\section{REFERENCES}

[1] Andrew Owens and Connelly Barnes "Camouflaging an Object from Many Viewpoints "1063-6919/14 \$31.00 @ 2014 IEEE DOI 10.1109/CVPR.2014.350

[2] KeGu, GuangtaoZhai, Xiaokang Yang, Wenjun Zhang, and Min Liu "STRUCTURAL SIMILARITY WEIGHTING FOR IMAGE QUALITY ASSESSMENT"

[3] Hu Jiang-hua, Qin Lei, Fu Tian-qi "Image Processing Based on Mathematical Morphology in Camouflage" 013.57978-0-7695-5050-3/13 \$26.00 @ 2013 IEEE DOI 10.1109/ICIG.2013.57

[4] Yusra A. Y. Al-Najjar, Dr. Der Chen Soong "Comparison of Image Quality Assessment: PSNR, HVS, SSIM, UIQI" International Journal of Scientific \& Engineering Research, Volume 3, Issue 8, August-2012 ISSN 2229-5518

[5] Marina Lukashevich, RaufSadykhov BSUIR, Minsk, Belarus "Texture Analysis: Algorithm for Texture Teatures Computation" IV International Conference "Problems of Cybernetics and Informatics" (PCI'2012), September 12-14, 2012

[6] ArashAbdiHejrandoost, Reza Safabakhsh "Thinning Based Multipurpose Camouflage Pattern Design " 978-14577-1535-8/11/\$26.00 @2011 IEEE

[7] Song Liming and GengWeidong " A new Camouflage Texture Evaluation Method based on WSSIM and Nature Image Features"978-1-4244-7874-3/10/\$26.00@2010

[8] Andrzej Materka and Michal Strzelecki "Texture Analysis Methods - A Review" 